\title{
Application of Modified ART1 Neural Network in Aero-engine Fault Diagnosis
}

\author{
Wang Hong-li, Xu Bing, Zheng Yuan, Xue Xue-dong, Cheng Kan \\ Dept. of 5, Wuhan Mechanical Technology, Wuhan 430075, China \\ email:1712178221@qq.com.cn
}

\begin{abstract}
Keywords: Aero-Engine; Adaptive Resonance Theory (ART) Network; Fault Diagnosis; Quadratic-estimation Four-circumscription
\end{abstract}

\begin{abstract}
According to principles of aero-engine fault diagnosis and flight data,this paper introduces a fault diagnosis method based on reformative ART1 neural network.After data pretreatment,it uses four-circumscription and quadratic-estimation to build fault codes and eliminate inveracious fault,and also ameliorate input layer of ART1 network.So this network can diagnoses not only gusty fault,but also gradual fault.It is proved that this method has high precision,strong restrain against noises, and low rate of inveracious alarms.
\end{abstract}

\section{Foreword}

With in-depth development in comprehensive integrated control of flight-propeller system for modern airplane, especially battle plane, flight data play a bigger role than ever. By availing and making full use of information on operating condition of airplane reflected by flight data, potential airplane fault can be discovered and removed in early stage with favorable safety reliability and redundancy for longer lifecycle of airplane and aero-engine on board.

However, due to impact from external foul flight environment, aging of relevant sensors, as well as causes such as random noise interference in fight data recording system and etc, certain error exists with flight data. To deal with it, a set of fault code extracting method is designed herein, and modified ART1 neural network is applied for fault diagnosis. ART1 neural network is highly effective for pinpoint and real-time diagnosis of accidental fault with aero-engine, together with foresighted forecast function for progressive fault.

\section{Introduction of ART1 Neural Network}

Adaptive Resonance Theory (ART) is a type of intensive learning and memory neural network developed by A. Carpenter from America, of which ART1 neural network functions to binary data processing. As shown in Figure 1, it's mainly a combination of two tiers of nerve cell and controlling signals. The network tier from input vector $P$ to output vector $A$ is called tier $\mathrm{R}$ (Recognition), a competition network; the network tier from output vector $A$ back to input vector $P$ is called tier $\mathrm{C}($ Comparison), an external network; tiers $\mathrm{R}$ and $\mathrm{C}$ respectively receive signals from three sources, that tier $\mathrm{R}$ receives output signal after competition of weighed input, controlling signal $G_{2}$ and reset signal Reset; tier $C$ receives input vector $P$, output (Return) signal form tier $\mathrm{R}$ and controlling signal $G_{1}$. Here only the functions of controlling signals $G_{1}, G_{2}$ and Reset are explained, as follows:

(1) $G_{1=1}$ only when all input signals $P$ are not 0 and all output signals in tier $\mathrm{R}$ are 0 , otherwise $G_{1}=0$ under all other conditions;

(2) $G_{2}=0$ only when all input signals $P$ not 0 , otherwise $G_{2}=1$;

(3) Under general conditions, Reset $=1$, when the sum of similarity of output in tiers $P$ and $\mathrm{C}$ is less than 0 (indicating this mode fails in meeting the requirement), Reset $=0$, and the network should be reset. 


\section{Fault Diagnosis Process}

Data Preprocessing.At first, initial filtering of flight data with Butterworth digital low-passing filter to eliminate outlier and bad value, so as to remove random noise interference in flight data recording system.

Establishment of Fault Code.ART1 network only functions to binary (or bipolar) data processing, thus data format needs to be converted to binary data representing data characteristics; flight data can be converted to binary data by setting of threshold based on indexes of parameters. During data conversion, 4 thresholds should be set respectively for all airplane parts according to performance indexes, namely, min. $P_{\min }$, min. normal $P_{\text {legal_min }}$, max. $P_{\max }$ and max. normal $P_{\text {legal_max }}$, here it's called “ 4 thresholds method”; definitions relating to these data are as follows:

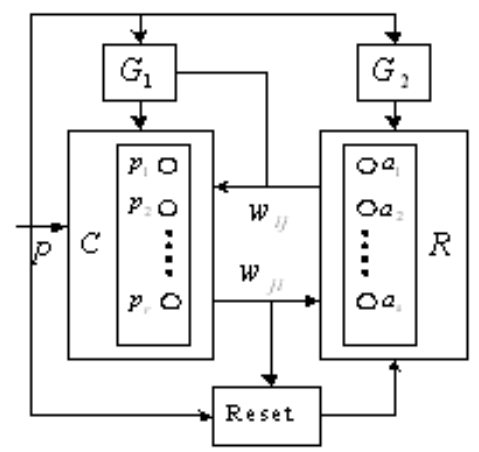

Figure 1 Structural Diagram of ART1 Network

Definition 2.1: of flight data, those values within the range of $P_{\text {legal_min }}$ and $P_{\text {legal_max }}$ are called normal data; and values within the range of $P_{\min }$ and $P_{\text {legal_min }}$ or $P_{\text {legal_max }}$ and $P_{\max }$ are called pseudo-fault data; both normal data and pseudo-fault data are called "legitimate" data.

Definition 2.2: of flight data, those values within the range of $-\infty$ and $P_{\min }$ or $P_{\max }$ and $+\infty$ are called burr data, also called "illegitimate" data.

So, according to "4 thresholds method", flight data link is divided into three types of data area of different characteristics, namely, normal data area, "legitimate" data area and "illegitimate" data area.

Processing of pseudo-fault data and burr data is the most crucial step to ensure accurate fault diagnosis. For burr data, in order to maintain continuous data recording, we convert them to "legitimate" pseudo value from "legitimate" data on both ends of burr data area, and the particular method of such conversion is to establish following equation on the principle of equivalent power of original data and pseudo value:

$$
\sum_{i=1}^{k}\left(P_{i}-P_{i-1}\right)^{2} \Delta T=\sum_{i=1}^{k}\left(P_{i}^{\prime}-P_{i-1}^{\prime}\right)^{2} \Delta T^{\prime}
$$

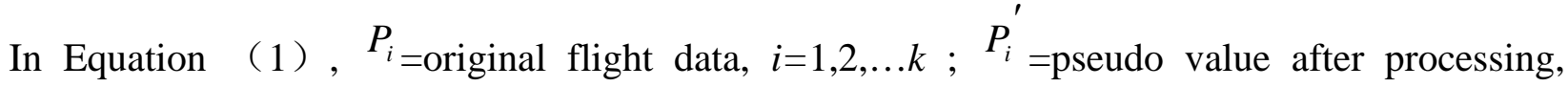
$i=1,2, \ldots k ; \Delta T$ and $\Delta T^{\prime}$ refer to interval between data recordings; $k=$ data length (Number) needs processing.

Equation (1) has two symmetric solutions on time axis, of which the solution at original data side is pseudo value.Pseudo value processing by equivalent power not only retains effective data information representing actual variation of aero-engine parameters while prevents omission in the reporting of aero-engine fault, but also eliminates the possibility of reporting of pseudo aero-engine fault as a result of the impact from foul flight environment and accidental fault of sensor.

However, if accidental data occur in "legitimate" area, above-mentioned "burr" data processing does not work, thus "pseudo fault" data within "legitimate" data area should be judged to determine whether they're fault data, here it is called "secondary judgment". 
Secondary judgment is made based on the algorithm of the duration of setting condition; duration of setting condition $n$ ( $\mathrm{n}$ is a time unit, which is generally taken to $1 \mathrm{~s} \sim 6 \mathrm{~s}$, depending on dynamic time characteristics of various parts and sensors ) ; if the length of pseudo fault data link is not less than $\mathrm{n}$ time unit, it's deemed as fault data; take arithmetic mean of fault related parameters within the range of $n$ as fault characteristic data, and remaining parameters are reset; if the length of pseudo fault data link is less than n time unit, it's deemed as pseudo fault data without fault data processing.

Still, some problems exist against extraction of fault data, for instance, one set of parameters representing the fault, and in case of occurrence of such fault, numerical values of some parameters vary within normal data area, for which case-based judgment should be made for this type of parameters during extraction of fault data; if these parameters vary within the range of representing a given fault phenomenon, and parametric data correlating to the same fault, such parameters are fault data; otherwise, these parameters are also pseudo fault data.

According to above principle, conversion function $f(x)$ can be established to form binary code; $f(x)$ is defined as follows:

$$
f(x)= \begin{cases}1, & x=\text { fault data } \\ 0, & x \mathrm{x}=\text { non }- \text { fault dat }\end{cases}
$$

\section{Method for Modifying ART1 Neural Network}

Due to some progressive faults of parts after aging or wearing, the results of above-mentioned secondary judgment belong to normal data, but they have evident fault tendency and potential risk which must be cautioned. In order to identify this type of potential faults, ART1 neural network is modified as follows:

Recode input fault code of the network according to following rule:

$$
\begin{aligned}
& f_{\Sigma i}(x)=\sum_{k=1}^{3} w_{k} \overline{f_{i-k}(x)}+f_{i}(x) \\
& \left\{\begin{array}{l}
\overline{f_{i}(x)}=0, \text { 当 } f_{\Sigma i}(x) \leq \omega \text { 时; } \\
\overline{f_{i}(x)}=1, \text { 当 } f_{\Sigma i}(x) \geq \omega \text { 时; }
\end{array}\right.
\end{aligned}
$$

when $\left|f_{\Sigma i}(x) \leq \omega\right|, \overline{f_{i}(x)}=0$; when $\mid f_{\Sigma i}(x) \geq \omega, \overline{f_{i}(x)}=1$

In Formula (3) and (4), $w_{k}=$ forgetting factor; $0 \leq w_{k} \leq 1, \overline{f_{i-k}(x)}=$ fault code during $k$ time; $f_{\Sigma i}(x)=$ weight integral effect of fault code; $f_{i}(x)$ means current fault code formed from Formula (2); $\overline{f_{i}(x)}=$ current recoded fault code; $\omega=$ fault criteria threshold, which is generally within $0.5 \sim 0.75$.

$\overline{f_{i}(x)}$, as input tier of ART1 neural network, has no impact on fault diagnosis principle mentioned above, rather, it takes into account time accumulation effect of fault potential and increases diagnosis sensitivity of progressive fault. In Formula (4), selection of forgetting factor $w_{k}$ and threshold $\omega$ is critical. Of three forgetting factors, the value earlier in time should be set as smaller value; excessively low $\omega$ could lead to false report, while excessively high $\omega$ could lead to lower sensitivity. Anyway, selection of both parameters has major impact on diagnosis outcome, and determination should be made based on calibration result or expertise.

\section{Algorithm Process and Outcome of Simulation Experiment}

Whole algorithm process is as shown in Flow Chart 2; for flight data after preprocessing, conversion to binary fault code should be conducted according to threshold, then these data are used as input for modified ART1 neural network after initial judgment of data processing of burr data, repeat training before selecting appropriate similarity to ensure lowest false diagnosis rate, and 
diagnosis outcome is obtained through network training.

Recorded flight data for a given type of aero-engine are selected to determine following 3 types of fault, including high-pressure over-speed of aero-engine; low-pressure over-speed of aero-engine; over-temperature of aero-engine, with four relevant parameters of speed of aero-engine HP rotor, speed of aero-engine LP rotor, exhaust temperature and movement of throttle lever.

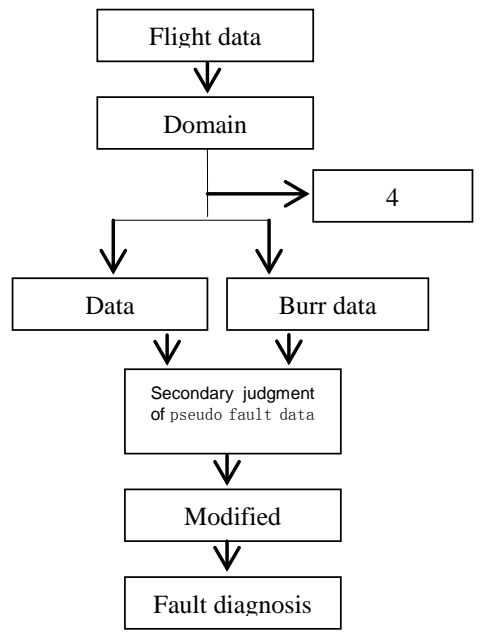

Figure 2-Algorithm Flow Chart

Take state holding to 4 time units, network similarity of $0.85, w_{k}=[0.05,0.03,0.065], \omega=0.55$; Establish fault code according to the method described herein.

In Table 1, for the fault of aero-engine over-temperature, both HP speed and LP speed are within normal range, due to exhaust temperature is used as fault data, and both HP speed and LP speed represent variance within the range of fault, they're also deemed as fault data. It's seen from Table 2 that classification results are correct.

Add in one set of fault data $\mathrm{E}$, fault codes after conversion are consistent with $\mathrm{B}$, and simulate one type of new fault codes $F=\left[\begin{array}{llll}1 & 1 & 1 & 1\end{array}\right]$, which a group of progressive faults.

It's obvious from Table 3 that, $\mathrm{E}$ are data by adding strong noise interference in B fault data; original diagnosis outcome of the network remains unchanged, and one group of new fault code $\mathrm{F}$ is created; the outcome indicates that (1) one new type of fault is generated in the network without impact on the determined faults; and (2) the network has strong anti-jamming capacity.

Table1-Fault Codes

\begin{tabular}{|c|c|}
\hline Type of fault & Outcome \\
\hline Over-speed of HP speed & B \\
\hline Over-speed of LP speed & A \\
\hline Over-temperature of aero-engine & C \\
\hline
\end{tabular}

Table 2-Classification Results

\begin{tabular}{|r|r|r|c|c|}
\hline $\begin{array}{c}\text { Type of } \\
\text { fault }\end{array}$ & $\begin{array}{r}\text { HP rotor } \\
\text { speed }\end{array}$ & $\begin{array}{r}\text { LP rotor } \\
\text { speed }\end{array}$ & $\begin{array}{c}\text { Exhaust } \\
\text { temperature }\end{array}$ & $\begin{array}{c}\text { Movement } \\
\text { throttle lever }\end{array}$ \\
\hline $\mathrm{B}$ & 1 & 0 & 0 & 1 \\
\hline $\mathrm{A}$ & 0 & 1 & 0 & 1 \\
\hline $\mathrm{C}$ & 1 & 1 & 1 & 0 \\
\hline
\end{tabular}

Table 3-Diagnosis Outcome

\begin{tabular}{|c|c|}
\hline Type of fault & Outcome \\
\hline Over-speed of HP speed & B,E \\
\hline Over-speed of LP speed & A \\
\hline Over-temperature of aero-engine & C \\
\hline Progressive fault & F \\
\hline
\end{tabular}




\section{Conclusion}

Preprocessing of flight data improves capacity of random interference resistance by diagnosis system. Modified ART1 neural network has powerful learning and memory functions; 4 thresholds method and the method for establishment of fault code by means of secondary judgment increases accuracy for collecting fault data; modifying input tier of ART1 neural network enhances its ability for identification and diagnosis of accidental fault and progressive fault. Experiment indicates that this method brings high precision, strong suppression of measureable noise, effective extraction of various data characteristics from flight data containing intense interference signal, this method is suitable for fault diagnosis, and it has remarkable practical value and engineering significance in improving the capacity for state monitoring and fault diagnosis of aero-engine.

\section{References}

[1] Li Hong and Wu Yiding, A Methodological Study on Fault Diagnosis of Aero-engine by ART1 Neural Network [J], Proceedings of the Chinese Society of Universities for Electric Power System and Automation, 2003.

[2] Fu Fan and Zhang Zonglin, Neural Network Applied to Fault Diagnosis and Method of Expert System [J], Journal of Northwest University(Natural Science Edition), 2003,146(Supplement): 94-95.

[3] GOMM J.Online Learning for Fault Classification Using an Adaptive Neuro-Fuzzy Network[A]. Proc of IFAC World Congress,1996.175-180.

[4] Cong Shuang, Theory and Application of MATLAB Toolbox Oriented Neural Network(2 $2^{\text {nd }}$ Edition)[M], Press of University of Science and Technology of Chin, Hefei, May 2003

[5] Li Renhou, Intelligent Control Theory \& Method [M], ; Xi'an: Xi'an University of Electronic Science and Technology Press, Oct 1999 